\title{
Factors affecting prognosis of status epilepticus among patients presenting to a tertiary care hospital
}

\author{
Wajid Jawaid', Muhammad Irfan², Sundus Mehtab Shafee ${ }^{3}$, \\ Sidra Javed Barry ${ }^{4}$, Sayed Mustafa Mahmood Shah ${ }^{5}$, Naila Shahbaz
}

\begin{abstract}
Objectives: This study aimed to evaluate the etiology, outcomes and prognostic factors associated with status epilepticus (SE) admissions in Neurology Department of a tertiary care hospital.

Methods: A retrospective review was performed on all SE admissions at Dr. Ruth K.M. Pfau Civil Hospital Karachi over a five-year period from July 2015 to June 2020. Demographic, clinical, and etiological factors were investigated for prognostic value. Statistical tests were applied to determine significant prognostic factors. A five percent significance level was used.

Results: A total of 176 patients were included in the study. Mortality was reported in 22 cases (12.5\%) and morbidity at six months was observed in 44 cases $(25.0 \%)$. Male gender, previous history of SE, prolonged seizure duration, and late presentation to hospital were significantly associated with mortality $(p<0.05)$. De novo cases of SE tended to be older $(p=0.048)$ and were associated with morbidity at followup $(p=0.000)$. The most common causes of epilepsy in our patients with SE were CNS infections $(n=54)$ and Idiopathic epilepsy $(n=34)$. Non-compliance to medicines/under-dosing was the most common provocative factor $(n=68)$. Acute symptomatic causes of SE were more likely to be associated with greater morbidity $(p=0.000)$. Refractory and super-refractory SE were strongly associated with higher mortality $(p=0.000)$. A longer duration of hospital stay was associated with higher morbidity $(\mathrm{p}=0.000)$.

Conclusion: Male gender, poor control of seizures, CNS infections, prolonged seizures, delayed hospital arrival and refractory/super-refractory status epilepticus were key determinants of mortality in our setting. Previous history of status epilepticus, and acute and symptomatic etiologies were associated with higher morbidity.
\end{abstract}

KEYWORDS: Status epilepticus, Prognostic factors, Mortality, Morbidity, Refractory, Super-refractory.

doi: https://doi.org/10.12669/pjms.38.1.5195

How to cite this:

Jawaid W, Irfan M, Shafee SM, Barry SJ, Shah SMM, Shahbaz N. Factors affecting prognosis of status epilepticus among patients presenting to a tertiary care hospital. Pak J Med Sci. 2022;38(1):16-22. doi: https://doi.org/10.12669/pjms.38.1.5195

This is an Open Access article distributed under the terms of the Creative Commons Attribution License (http://creativecommons.org/licenses/by/3.0), which permits unrestricted use, distribution, and reproduction in any medium, provided the original work is properly cited.

Wajid Jawaid, FCPS Neurology,

Muhammad Irfan, FCPS Neurology,

Sundus Mehtab Shafee, MBBS.

Sidra Javed Barry, MBBS.

Sayed Mustafa Mahmood Shah, MBBS.

Naila Shahbaz, FCPS Neurology,

1-6: Department of Neurology,

Dow University of Health Sciences \&

Dr. Ruth K. M Pfau Civil Hospital, Karachi, Pakistan.

Correspondence:

Dr. Wajid Jawaid,

Department of Neurology,

Dow University of Health Sciences \&

Dr. Ruth K. M Pfau Civil Hospital, Karachi, Pakistan.

E-mail: wajid.jawaid@outlook.com

* Received for Publication:

August 16, 2021

* Revision Received:

* Revision Accepted:

November 30, 2021

\section{INTRODUCTION}

Status epilepticus (SE) is a neurological emergency characterized by continuous seizures for more than five minutes or recurrent seizure activity for more than five minutes without recovery between seizures. ${ }^{1}$ The estimated global incidence of SE ranges from 1.29 to $73.7 / 100,000$ with wide variation across location and type of study. ${ }^{2}$ Within developing countries, the pooled crude annual incidence is reported as 13.8 per $100,000 .^{3,4}$ Despite advances in understanding the clinical course of SE and optimal management, short-term mortality remains as high as 3-40\% depending on the center and type of SE encountered. ${ }^{5}$ 
An approach to further delineate components of SE with a bearing on clinical course and management has been made by the International League Against Epilepsy (ILAE) by distinguishing separate axes for SE on the basis of semiology, EEG correlates, etiology, and age at onset. ${ }^{6}$ This has fueled scope for further study into the broad array of prognostic factors that may aid in identifying SE patients considered high-risk and guide optimal treatment decisions. Matching the degree of treatment and intensity of care to the severity of SE encountered will not only streamline delivery of care, but will avoid the risks carried by overtreatment of patients with milder disease. ${ }^{7}$

There is a paucity of data relaying the epidemiology, prognosis and outcomes of SE presenting within South-Asia. This is particularly true of Pakistan where only a couple of pediatric studies on SE have been published. ${ }^{8,9}$ This has significant implications for the development of a sound clinical approach given the unique demands of the region. We aimed to fill this gap by conducting a retrospective observational study of SE admissions over a five-year period to delineate the demographic, etiological, and clinical characteristics of SE patients presenting to a tertiary care hospital in Karachi, Pakistan. The outcomes of interest included in-hospital mortality and 6-month morbidity, for which key prognostic variables were investigated.

\section{METHODS}

Study design and duration: A retrospective observational study was conducted at the Department of Neurology, Dr. Ruth K.M. Pfau Civil Hospital Karachi. All patients admitted in Neurology ICU with status epilepticus for a fiveyear period from July 2015 to June 2020 were included.

Patient Management: Patients were managed under a standardized treatment protocol in keeping with current treatment algorithms, which included initial administration of IV benzodiazepine (diazepam/midazolam), followed by stepwise administration of IV anti-seizure drug (phenytoin/valproic acid/levetiracetam) and IV anesthetic agent (midazolam infusion/propofol infusion) as needed. Administered doses of these drugs were variable, based on the patients' weight and response to the drug. Airway was secured; continuous monitoring was instituted for all patients.
Data collection: The department's record was used to collect data on demographic variables such as age, gender and residence of the patients. Clinical course, in-hospital management and response to therapy were also recorded from the data. Follow-up assessment of sequelae and patients' progress over the following six months completed our data collection from the records.

Etiologies of SE were divided temporally as acute or chronic, and considered separately as cryptogenic (having no identifiable causative factor) or symptomatic (occurring due to another distinct disease entity) ${ }^{10}$ SE was classified by semiology as per the recent ILAE classification and guidelines. ${ }^{6}$

Inclusion criteria: Patients aged 12 years or more who were admitted with status epilepticus in the study setting during the study period were included.

Exclusion criteria: Patients who were initially managed in some other department or the ones who were taken out against medical advice before completion of treatment were excluded.

Definitions: Status epilepticus was defined as continuous seizure activity for at least five minutes or recurrent seizures for more than five minutes without recovery between seizures. Refractory status epilepticus was defined as SE episodes not controlled by first-line and second line AED, thus requiring IV anesthetics. SE remaining for 24 hours or more following anesthetic administration was defined as superrefractory SE. ${ }^{11}$ Morbidity in this study was considered when patients developed cognitive impairment, motor deficits or active epilepsy (alone or in combination) as a complication of SE. Scar epilepsy refers to delayed presentation of epilepsy as a sequelae of previous cerebral insult like tumor, trauma, surgery, stroke etc.

Statistical Analysis: Statistical Analysis was performed using IBMSPSS version 21. Descriptive statistics and frequencies were calculated. The normal distribution of the variables was tested by one sample Kolmogorov-Smirnov test. Chisquared test with 95\% confidence interval was used to compare categorical variables. Analysis of variable with small cell scores was computed using Fisher's Exact Test or Likelihood Ratio, as appropriate. Non-parametric tests were used to compare differences in continuous variables across groups. A five percent significance level was used throughout the study. 




Fig.1: Prognosis among study population.

Ethical Considerations: The study was approved by the Institutional Review Board of Dow University of Health Sciences on $15^{\text {th }}$ September, 2021 [IRB-2100/DUHS/Approval/2021/523].

\section{RESULTS}

There was a total of 176 admitted patients with SE during the study period. Mortality was reported in 22 cases $(12.5 \%)$ and morbidity was reported in 44 cases $(25.0 \%)$. Further grouping of different morbid sequelae with their frequency is represented in Fig.1.

The median age of our study population was 24 (IQR=15.0-31.75).Gender and residential information is given in Fig.2. Male gender was strongly associated with mortality $(p=0.002)$ but not morbidity $(\mathrm{p}=0.356)$. A majority had not received any formal education (60\%). A previous history of status epileptic attacks was significantly associated with mortality $(\mathrm{p}=0.004)$. Other sociodemographic variables were not significantly associated with mortality or morbidity $(p>0.05)$ (Table-I).

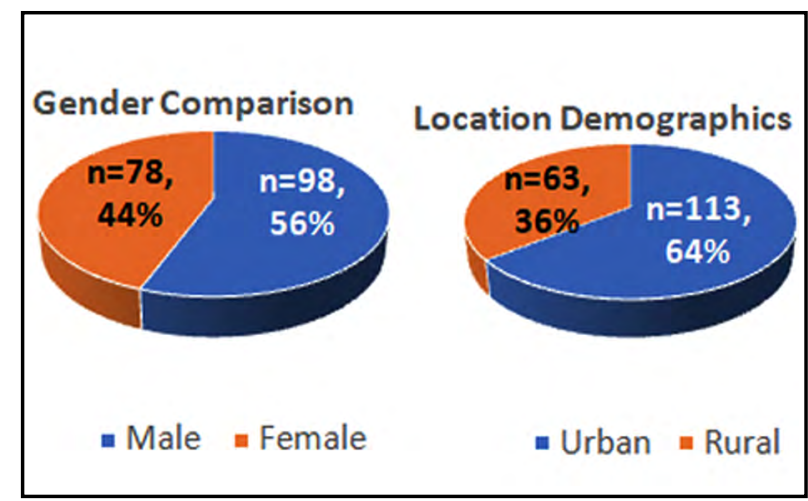

Fig.2: Socio-demographic characteristics of study population.

The most common causes of epilepsy in our patients with SE were found to be CNS infections $(n=54)$ and Idiopathic epilepsy $(n=34)$ (Fig.3). The etiological characteristics of SE are outlined in Table-II. Most cases of SE occurred after a previous history of epilepsy $(n=140,79.5 \%)$. De novo cases of SE were associated with greater morbidity $(p=0.00)$, but not with greater mortality $(p=0.862)$. Older age was significantly associated

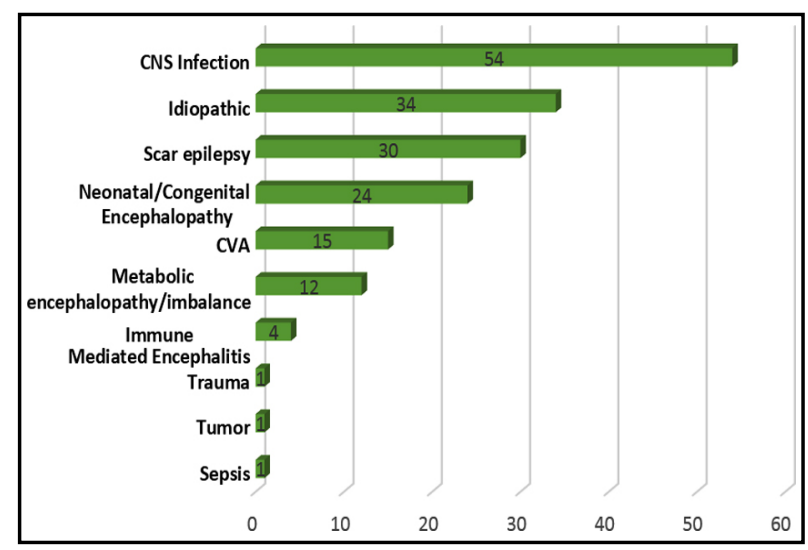

Fig.3: Etiology of epilepsy in study population.

Table-I: Socio-demographic characteristics of study population.

\begin{tabular}{lccc}
\hline Characteristics & $\begin{array}{c}\text { Frequency } \\
n(\%)\end{array}$ & $\begin{array}{c}\text { Association with Morbidity } \\
(p \text {-Value })\end{array}$ & $\begin{array}{c}\text { Association with Mortality } \\
(p \text {-Value })\end{array}$ \\
\hline Males & $98(55.7)$ & 0.356 & 0.002 \\
Uneducated & $105(59.6)$ & & \\
Primary education & $41(23.2)$ & 0.110 & 0.791 \\
Secondary education & $28(16.0)$ & & \\
Graduated & $2(1.1)$ & 0.402 & 0.116 \\
Family history of epilepsy & $9(5.1)$ & 0.808 & 0.004 \\
History of status epilepticus & $24(13.8)$ & & \\
\hline
\end{tabular}


Wajid Jawaid et al.

Table-II: Etiological characteristics of study population.

\begin{tabular}{llcccc}
\hline Characteristics & & $(n)$ & $(\%)$ & $\begin{array}{c}\text { Association with } \\
\text { Morbidity(p-Value) }\end{array}$ & $\begin{array}{c}\text { Association with } \\
\text { Mortality }(p \text {-Value })\end{array}$ \\
\hline \multirow{2}{*}{$\begin{array}{l}\text { History of prior } \\
\text { seizures }\end{array}$} & De novo status epilepticus & 36 & 20.4 & 0.00 & 0.862 \\
& History of epilepsy & 140 & 79.5 & $>0.05$ & $>0.05$ \\
Type of etiology & Cryptogenic & 38 & 21.5 & $>0.05$ & 0.108 \\
\multirow{2}{*}{$\begin{array}{l}\text { Time course of } \\
\text { etiology }\end{array}$} & Acute & 138 & 78.4 & 0.009 & 0.171 \\
\hline
\end{tabular}

with a presentation of de novo SE (0.048). Symptomatic causes of SE were associated with greater morbidity as compared to cryptogenic SE $(p=0.009)$; no such association was found with respect to mortality $(p=0.171)$. Acute causes of $S E$ were similarly associated with higher likelihood of morbidity $(p=0.00)$, but not with higher mortality $(p=0.137)$. A longer duration of hospital stay was associated with the occurrence of SE due to an acute etiology $(\mathrm{p}=0.000)$ and symptomatic SE (0.003).

Cases of SE which were precipitated following a defined provocative factor are summarized in Table-III. Non-compliance to medicines/underdosing was the most common provocative factor $(n=68,38.6 \%)$, followed by infective etiologies $(\mathrm{n}=55,31.3 \%)$. Highest mortality and morbidity rate were seen in infective etiology $(54.5 \%$ and $27.2 \%$ respectively (Table-III).

The clinical characteristics of SE patients are illustrated in Fig-4. SE with prominent motor phenomenon (generalized tonic clonic seizures) was the most common type $(87.9 \%)$. Most patients presenting with SE reported poor seizure control in the preceding three months (58\%), which was associated with higher morbidity at followup $(p=0.000)$. Most cases of SE were found to be responsive to medical management $(85.9 \%)$, while the remaining were categorized as having refractory or super-refractory status epilepticus. Type of seizure in SE was not significantly associated with mortality nor morbidity ( $\mathrm{p}>0.05)$.

Table-III: Provocative factors associated with onset of status epilepticus.

\begin{tabular}{llll}
\hline Provocative Factor & $\begin{array}{l}\text { Frequency } \\
n(\%)(n=176)\end{array}$ & $\begin{array}{l}\text { Morbidity } \\
n(\%)(n=44)\end{array}$ & $\begin{array}{l}\text { Mortality } \\
n(\%)(n=22)\end{array}$ \\
\hline Noncompliant to Meds/ Under-dosed & $68(38.6)$ & $8(18.2)$ & $5(22.7)$ \\
Infective etiology & $55(31.3)$ & $12(27.2)$ & $12(54.5)$ \\
Unprovoked & $20(11.3)$ & $8(18.8)$ & $1(4.5)$ \\
Vascular event & $15(8.5)$ & $9(20.4)$ & $1(4.5)$ \\
Metabolic derangement & $12(6.8)$ & $5(11.3)$ & $2(9.0)$ \\
Immune-mediated & $4(2.2)$ & $1(2.2)$ & $0(0.0)$ \\
Trauma & $1(0.6)$ & $0(0.0)$ & $1(4.5)$ \\
Tumor & $1(0.6)$ & $1(2.2)$ & $0(0.0)$ \\
\hline
\end{tabular}

Table-IV: Temporal Profile of Seizures.

\begin{tabular}{lccc}
\hline Characteristics & $\begin{array}{c}\text { Median } \\
(\text { IQR) }\end{array}$ & $\begin{array}{c}\text { Association with } \\
\text { Morbidity }(p \text {-Value) }\end{array}$ & $\begin{array}{c}\text { Association with } \\
\text { Mortality }(p \text {-Value })\end{array}$ \\
\hline Seizure Duration & 60 minutes (30-65) & 0.842 & 0.00 \\
Hospital Stay & 7 days (4.25-10.75) & 0.00 & 0.115 \\
Time to Hospital Arrival & 45 minutes (30-120) & 0.712 & 0.001 \\
\hline
\end{tabular}




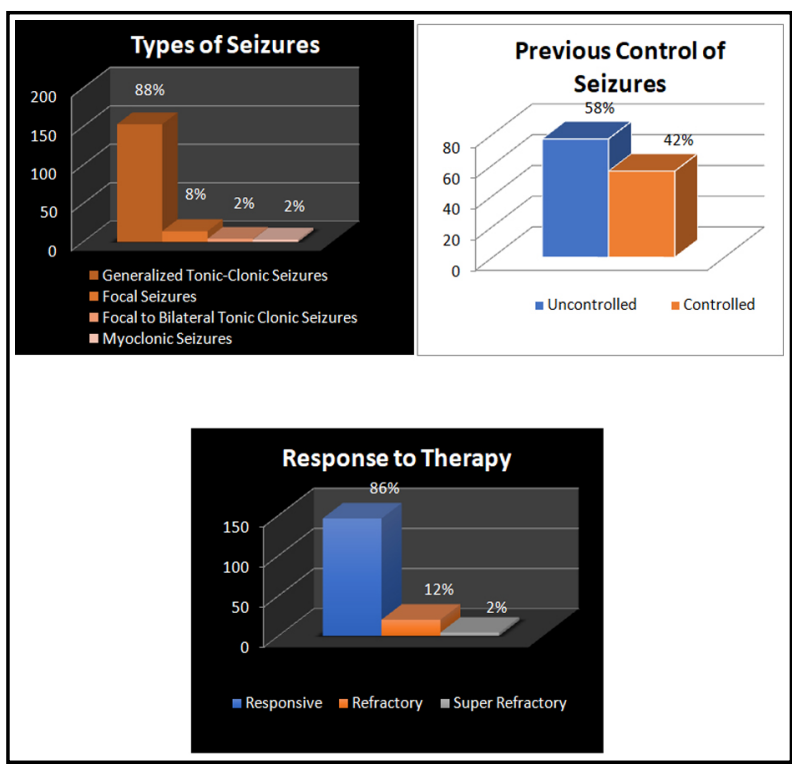

Fig.4: Clinical characteristics of seizures in SE patients.

Refractory and Super-refractory SE status was strongly associated with higher mortality $(\mathrm{p}=0.000)$.

The temporal profile of seizures are illustrated in Table-IV. The median hospital stay was seven days (IQR $=4.25-10.75)$. Patients with a prolonged seizure duration and longer interval to hospital presentation were associated with higher mortality $(p=0.000)$ and $(p=0.000)$ respectively.

\section{DISCUSSION}

Status epilepticus is the second most common neurological emergency. ${ }^{12}$ "Time is brain" is not only a cliché when it comes to this potentially devastating condition that has bearing on the patients and their families. ${ }^{13}$ When dealing with an adversary, it is of utmost importance to know the arsenal at their disposal. This is where this study plays its role by outlining not only the prognostic factors in SE, but also the clinical, etiological, and demographic profile of SE patients presenting in a tertiary care hospital in Karachi over a five-year period.

The case fatality rate observed in this retrospective cohort was $12.5 \%$. This is in line with the case fatality rate in developing countries of $15.6 \%$ as reported by a meta-analysis on the subject. $^{3}$ Our study reported many potential factors implicated in the prognosis of SE and the significance of the causative etiology in SE outcomes. Male gender was found to be significantly associated with higher mortality $(25.6 \%$ vs $19.4 \%$ in females). Multiple studies have documented a male preponderance in the occurrence of SE. ${ }^{14,15}$ Yet the effect of gender on outcomes remains unclear in the literature, with some studies reporting no effect $\mathrm{t}^{5,7,16}$, higher fatality rate in females, ${ }^{4}$ or greater mortality in males. ${ }^{17}$ It is clear that acute symptomatic etiologies are associated with worse outcomes in SE patients, and the higher incidence of such etiologies in men may explain the higher incidence in the literature, ${ }^{18}$ and the poorer outcome in this study.

Advancing age trended towards poorer functional outcomes in our study. The relationship of advancing age and poor outcomes in SE has been clearly documented, which has led to the incorporation of age into various prognostic scoring systems for SE including the status epilepticus severity score (STESS) and the Epidemiology-based Mortality Score in Status Epilepticus (EMSE). ${ }^{19,20}$

A longer duration of SE and longer interval to hospital presentation were found to be significantly associated with greater mortality in this study. In a recent review Alkhachroum et al., discussed multiple studies documenting the same. They emphasized that the effect of SE duration on outcomes was strongly influenced by the underlying etiology. ${ }^{18}$ Our hospital serves as a major tertiary-care referral center at a city and provincial level, therefore more than one-third of SE presentations we received were from rural centers. This throws light on lack of facilities in the rural areas necessitating referral to our hospital, ${ }^{21}$ thereby increasing the time to receive appropriate management due to distance and adversely affecting the outcomes. Further study into the prevalence of SE in rural settings and implications for timely care are therefore warranted.

De novo cases of SE and SE arising from a symptomatic disease process were positively associated with worse functional outcomes at six-month follow-up. De novo cases of SE were also associated with older age. These findings are consistent with those reported by Lui et al, from an 11-year retrospective study conducted in Hong Kong, ${ }^{22}$ and are likely attributable to the acute symptomatic etiologies which accompany de novo and symptomatic SE. Further analysis of the etiologies underlying presentations during our study period confirmed the same; CNS infection was more strongly associated with worse outcomes as compared to SE due to cryptogenic epilepsy or non-compliance to 
medicines $(p<0.005)$. A recent meta-analysis has also shown CNS infections to be an important cause of SE in the developing world, likely due to unsanitary conditions and inconsistent provision of healthcare. ${ }^{3}$ This has significant implications for the creation of a regional action plan in developing countries where proposals to address the burden of SE must address comprehensive infection-control practices in the region. Scar epilepsy was the third-most common etiology of epilepsy among our SE patients. The cerebral insults resulting in scar epilepsy are often result of a co-morbid condition like diabetes, hypertension, obesity or lifestyle factors like smoking, helmet-less bike riding etc.

Refractoriness of SE episodes was associated with mortality, whereas a longer duration of hospital stay was associated with poorer functional outcomes. A review by Nelson et al, noted that while the most common causes of SE may vary across various settings, the etiology of refractory SE appears to be similar to that of non-refractory SE. ${ }^{11}$ Our experience and analysis are in agreement with these observations. In the same review it is noted that super refractory SE may have a different etiological profile and commonly arises in the setting of encephalitis. ${ }^{11}$ This is especially alarming as a recent study from our centre found CNS infections as among the most common causes of symptomatic epilepsy in both pediatric and adult age group patients in our set-up. ${ }^{23}$ Among the four cases of super refractory SE in this study, three were secondary to CNS infections. The need of the hour is to prevent super-refractory status epilepticus by controlling the risk factors in the population, as mortality rate in these cases ranges from 30 to $50 \% .{ }^{24}$

Limitations of the study: This was a retrospective study confined to one tertiary care hospital. Moreover, our set-up lacks the resources for continuous EEG monitoring of patients under ICU care. Continuous EEG monitoring has been advocated as an effective means of early detection of epileptic activity in patients admitted in the ICU, particularly for non-convulsive seizures, and can allow for the prognostication of patients at the bedside. ${ }^{25}$

\section{CONCLUSION}

Prognosis of patients presenting with status epilepticus is influenced by socio-demographic and clinical variables. Male gender, poor control of seizures, CNS infections, prolonged seizures, delayed hospital arrival and refractory/ super-refractory status epilepticus were key determinants of mortality in our setting. Previous history of status epilepticus and cases with acute and symptomatic etiologies were associated with higher morbidity. Public awareness programs should be conducted to emphasize on modifiable risk factors including drug compliance and seizure control as these can alter outcome of patients. More local studies are required regarding prognostic factors to provide high sensitivity and specificity in predicting outcomes of status epilepticus.

Conflicts of Interest: None.

Source of Funding: None.

\section{REFERENCES}

1. Brophy GM, Bell R, Claassen J, Alldredge B, Bleck $\mathrm{TP}$, Glauser T, et al. Guidelines for the evaluation and management of status epilepticus. Neurocrit Care. 2012;17(1):3-23. doi: 10.1007/s12028-012-9695-Z

2. Leitinger $M$, Trinka E, Zimmermann G, Granbichler CA, Kobulashvili T, Siebert U. Epidemiology of status epilepticus in adults: Apples, pears, and oranges - A Critical Review. Epilepsy Behav. 2020;103(Pt A):106720. doi: 10.1111/epi.14607

3. Lv RJ, Wang Q, Cui T, Zhu F, Shao XQ. Status epilepticusrelated etiology, incidence and mortality: A metaanalysis. Epilepsy Res. 2017;136:12-17. doi: 10.1016/j. eplepsyres.2017.07.006

4. Leitinger M, Trinka E, Giovannini G, Zimmermann G, Florea C, Rohracher A, et al. Epidemiology of status epilepticus in adults: A population-based study on incidence, causes, and outcomes. Epilepsia. 2019;60(1):5362. doi: 10.1111/epi.14607

5. Stelzer FG, Bustamante Gde O, Sander H, Sakamoto AC, Fernandes RM. Short-term mortality and prognostic factors related to status epilepticus. Arquivos De NeuroPsiquiatria. 2015;73(8):670-675. doi: 10.1590/0004282X20150082

6. Trinka E, Cock H, Hesdorffer D, Rossetti AO, Scheffer IE, Shinnar S, et al. A definition and classification of status epilepticus--Report of the ILAE Task Force on Classification of Status Epilepticus. Epilepsia. 2015;56(10):1515-1523. doi: 10.1111/epi.13121

7. Atmaca MM, Bebek N, Baykan B, Gokyigit A, Gurses C. Predictors of outcomes and refractoriness in status epilepticus: A prospective study. Epilepsy Behav. 2017;75:158-164. doi: 10.1016/j.yebeh.2017.07.046

8. Uzair M, Ibrahim A, Zafar F, Sultan T. Etiology and outcomes of convulsive status epilepticus in children. Pak J Med Sci. 2019;35(3):620-623. doi: 10.12669/ pjms.35.3.120

9. Ibrahim SH, Yezdan MA, Nizami SQ. Status epilepticus in children: a five-year experience at Aga Khan University Hospital. J Pak Med Assoc. 2003;53(12):597-599.

10. Betjemann JP, Lowenstein DH. Status epilepticus in adults. Lancet Neurol. 2015;14(6):615-624. doi: 10.1016/S14744422(15)00042-3 
11. Nelson SE, Varelas PN. Status Epilepticus, Refractory Status Epilepticus, and Super-refractory Status Epilepticus. Continuum (Minneapolis, Minn). 2018;24(6):1683-1707. doi: 10.1212/CON.0000000000000668

12. Janszky J, Bone B, Horvath R, Suto Z, Szapary L, Juhos V, et al. Status epilepticus 2020. Orv Hetil. 2020;161(42):17791786. doi: $10.1556 / 650.2020 .31908$

13. Der-Nigoghossian C, Rubinos C, Alkhachroum A, Claassen J. Status epilepticus - time is brain and treatment considerations. Curr Opin Crit Care. 2019;25(6):638-646. doi: $10.1097 /$ mcc.0000000000000661

14. Halawa EF, Draz I, Ahmed D, Shaheen HA. Predictors of Outcome of Convulsive Status Epilepticus Among an Egyptian Pediatric Tertiary Hospital. J Child Neurol. 2015;30(13):1736-1742. doi: 10.1177/0883073815579706

15. Hesdorffer DC, Logroscino G, Cascino G, Annegers JF, Hauser WA. Incidence of status epilepticus in Rochester, Minnesota, 1965-1984. Neurology. 1998;50(3):735-741. doi: 10.1212/wnl.50.3.735

16. Power KN, Gramstad A, Gilhus NE, Engelsen BA. Prognostic factors of status epilepticus in adults. Epileptic Disord. 2016;18(3):297-304. doi: 10.1684/epd.2016.0855

17. Dham BS, Hunter K, Rincon F. The epidemiology of status epilepticus in the United States. Neurocrit Care. 2014;20(3):476-483. doi: 10.1007/s12028-013-9935-x

18. Alkhachroum A, Der-Nigoghossian CA, Rubinos C, Claassen J. Markers in Status Epilepticus Prognosis. J Clin Neurophysiol. 2020;37(5):422-428. doi: 10.1097/ WNP.0000000000000761

19. Rossetti AO, Logroscino G, Milligan TA, Michaelides C, Ruffieux C, Bromfield EB. Status Epilepticus Severity Score (STESS): A tool to orient early treatment strategy. J Neurol. 2008;255(10):1561-1566. doi:10.1007/s00415-008-0989-1

20. Leitinger M, Höller Y, Kalss G, Rohracher A, Novak HF, Höfler J, et al. Epidemiology-based mortality score in status epilepticus (EMSE). Neurocrit Care. 2015;22(2):273282. doi: $10.1007 /$ s12028-014-0080-y

21. Kurji Z, Premani ZS, Mithani Y. Analysis of the Health Care System of Pakistan: Lessons Learnt and Way Forward. J Ayub Med Coll Abbottabad. 2016;28(3):601-604.
22. Lui HK, Hui KF, Fong WC, Ip CT, Lui HT. De novo status epilepticus is associated with adverse outcome: An 11-year retrospective study in Hong Kong. Seizure. 2016;40:42-45. doi:10.1016/j.seizure.2016.06.006

23. Jawaid W, Nisa Q, Umer SR, Barry SJ, Qureshi A, Shahbaz NN. Etiology and Types of Seizures in Patients Presenting to a Tertiary Care Hospital in Karachi: A Cross-Sectional Study. Cureus. 2020;12(7):e9194. doi: 10.7759/cureus.9194

24. Kirmani BF, Au K, Ayari L, John M, Shetty P, Delorenzo RJ. Super-Refractory Status Epilepticus: Prognosis and Recent Advances in Management. Aging Dis. 2021;12(4):10971119. doi: 10.14336/AD.2021.0302

25. Tiamkao S, Saybungkla P, Sirikarn P, Sawanyawisuth K, Integrated Epilepsy Research G. Predictors of longterm mortality in status epilepticus. Epilepsy Behav. 2018;84:114-117. doi: 10.1016/j.yebeh.2018.04.027

\section{Authors' Contribution:}

WJ: Conception and design, data acquisition, drafting and critical revision of the manuscript; responsible and accountable for the accuracy and integrity of the work.

MI: Design, data acquisition, critical revision of the manuscript.

SMS: Design, data acquisition, drafting the manuscript.

SJB: Design, data acquisition, drafting the manuscript.

SMMS: Data analysis and interpretation, drafting the manuscript.

NS: Conception and design, critical revision of the manuscript. 